ACTA UNIVERSITATIS CIBINIENSIS - TECHNICAL SERIES

Vol. LXVII 2015

\title{
FAULTS DETECTION AND LEVEL CONTROL IN COUPLED TANKS
}

\author{
MAICAN Camelia \\ Lecturer/Ph.D., Faculty of Automation, Computers and Electronics/Department of Automation and Electronics, \\ University of Craiova, Craiova, Romania, camelia@automation.ucv.ro
}

\begin{abstract}
This paper presents a mechanism for the detection and localization of faults in a plant, which is equipped with two coupled tanks, using residual methods. The water circuit consists of the upper and lower tanks connected through a system of pipes and valves. The controlled variable is the level in the upper tank. The level control system and the faults detection structure were developed under Matlab Simulink. The faults detection structure allows us to detect two faults that can occur in the plant, separately. The proposed method was theoretically developed and experimentally verified on the plant model.
\end{abstract}

Key words: fault detection; level control; residue

\section{Introduction}

A fault in a dynamical system can often be defined as a deviation from the nominal state in the system parameters or structure [1]. Fault detection is the indication that something is going wrong in the monitored system. It was proposed many different approaches to fault detection based either on the model of the system (analytical methods) [2] or on the knowledge about the system (heuristic methods) [3, 4, 5]. The laboratory plant can be the subject of a series of additive faults and disturbances. The faults and disturbances can appear in processing equipment (example: the breaking of a pipe in the tank) or in auxiliary equipments (example: faults of the actuators and the faults of the transducers). Faults can negatively influence the quality of the production or can damage the machinery or harm humans. Therefore the faults shall be quickly detected and eliminated. In order to detect a fault, we need a reference for the nominal plant operation. This reference can be generated from a plant mathematical model describing the relationship between plant inputs and outputs.

This system has the typical characteristics of tanks, pipelines and pumps used in many industries. In this case, the faults are: the pump is not running completely the command it receives or there may be an additional pressure loss and obturating the flowing section or additional pipeline breakings.

This paper is organized as follows: the system with two tanks is described in section 2, the mathematical model of the plant is presented in section 3, in section 4 is presented the implementation of the faults detection structure and the experimental results, the conclusions are presented in section 5.

\section{Description of the plant}

The plant is equipped with two tanks of identical quantity, a level transducer with ultrasounds mounted on the superior tank, the variable flow supply pump, local regulator for adjustment of the pumps flow, flow transducer to measure the output flow from the superior tank, and flow transducer to measure the pumps flow at water recalculating in the inferior tank [6, 7].

For the level measurement in the upper tank, the sensor is generating an ultrasonic wave, which is reflected by the water surface and received back by the sensor. The atmospheric air is used as propagation medium for the ultrasonic waves. The sensor output is proportional with the transit time of the ultrasonic wave between the sensor and the water surface and is generated by an electronic module connected to the sensor. The output signal of the sensor is a 4-20 mA current signal, for a variation of level between 0-500 $\mathrm{mm}[6]$. 
The liquid level control is achieved by changing the water pump flow, using the speed command of the driving motor. This is achieved by varying the supply voltage of the pump using the pump regulator, which is driven with a voltage signal in the range of 2-10 V DC.

The hydraulic system diagram of the plant is shown in Figure1 [7].

The state variables are:

$L_{1}=x_{1}$ the level of the tank1

$L_{2}=x_{2}$ the level of the tank2

The input variables are :

$U_{c}$ the command to adjust the level in the tank 1

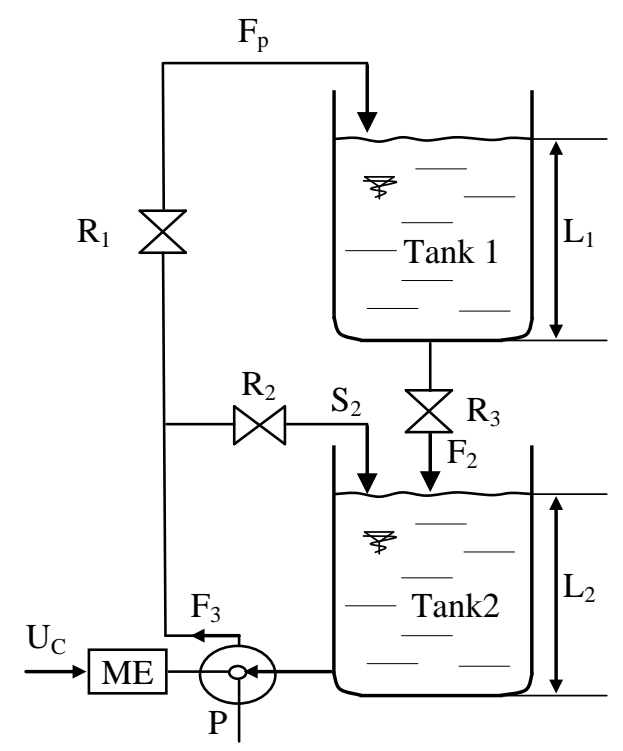

Figure 1: The hydraulic system diagram

The didactics plant allows the level control in the upper tank, using flow's command corresponding to the water's recirculation pump from the lower basin of the upper basin. The disturbance is the flow section of the pipe, between the two tanks.

\section{Mathematical models}

In order to implement the faults detection diagram it is necessary to determine the mathematical model of the plant as accurate as possible, to emphasize the influence of the faults and the disturbances on observable variables and then the reduction of the processing requirements.

The mathematical model of the system is determined using the mass balance equations [7]:

$$
\begin{aligned}
& \frac{d L_{1}}{d t}=\frac{F_{p}\left(U_{C}\right)-F_{2}\left(S_{2}, L_{1}\right)}{A_{1}}=\frac{k_{p} U_{C}-C S_{2} \sqrt{L_{1}}}{A_{1}} \\
& \frac{d L_{2}}{d t}=\frac{F_{2}\left(S_{2}, L_{1}\right)-F_{3}\left(U_{C}\right)}{A_{2}}=\frac{C S_{2} \sqrt{L_{1}}-k_{p} U_{C}}{A_{2}}
\end{aligned}
$$

Where $C$ is a coefficient depending on the viscosity of the fluid loss and sectional shape of the flowing, $S_{2}$ sectional area of the valve transitions between the two tanks, $A_{1}$ and $A_{2}$ cross-sectional area of the tank1, respectively tank2, $F_{p}$ is the inflow rate of tank1 and $F_{2}$ is the outflow rate from tank2.

In canonical form the mathematical model is:

$$
\begin{aligned}
& \frac{d L_{1}}{d t}=-\frac{C}{A_{1}} S_{2} \sqrt{L_{1}}+\frac{k_{P}}{A_{1}} U_{C} \\
& \frac{d L_{2}}{d t}=\frac{C}{A_{2}} S_{2} \sqrt{L_{1}}-\frac{k_{P}}{A_{2}} U_{C}
\end{aligned}
$$

In steady state:

$$
k_{p} U_{C 0}=C S_{20} \sqrt{L_{10}}=F_{20}
$$


By linearizing of the equations (3) and (4) around the steady state values, resulting linear form of the mathematical model (6) and (7), which contain the section variation $\Delta S_{2}$ which can occur only under fault conditions. Under normal conditions $\Delta S_{2}=0$.

$$
\begin{aligned}
& \Delta \dot{x}_{1}=-\frac{C S_{20}}{2 A_{1} \sqrt{L_{10}}} \Delta x_{1}+\frac{k_{p}}{A_{1}} \Delta U_{C}-\frac{C \sqrt{L_{10}}}{A_{1}} \Delta S_{2} \\
& \Delta \dot{x}_{2}=\frac{C S_{20}}{2 A_{2} \sqrt{L_{10}}} \Delta x_{1}-\frac{k_{p}}{A_{2}} \Delta U_{C}+\frac{C \sqrt{L_{10}}}{A_{2}} \Delta S_{2}
\end{aligned}
$$

Where: $\quad \Delta x_{1}=\Delta L_{1}=L_{1}-L_{10} ; \quad \Delta x_{2}=\Delta L_{2}=L_{2}-L_{20} ; \quad x_{10}=L_{10}$ and $x_{20}=L_{20}$

Replacing the coefficient $\mathrm{C}$ obtained from equation (5) and applying Laplace transform in zero initial conditions result:

$$
\begin{aligned}
& \Delta x_{1}(s)=\frac{1}{T s+1}\left[\frac{2 x_{10} k_{p}}{F_{20}} \Delta U_{C}(s)-\frac{2 x_{10}}{S_{20}} \Delta S_{2}(s)\right] \\
& \Delta x_{2}(s)=\frac{A_{1} / A_{2}}{T s+1}\left[-\frac{2 x_{10} k_{p}}{F_{20}} \Delta U_{C}(s)+\frac{2 x_{10}}{S_{20}} \Delta S_{2}(s)\right]
\end{aligned}
$$

Where: $\quad T=\frac{2 A_{1} x_{10}}{F_{20}}$

\section{The implementation of the faults detection structure}

The method is based on the difference between the real plant with fault and the model without the fault. The input model applies the same command variables as the real plant, so this difference (residue) is influenced both by the faults and the disturbances.

We will analyze the following faults which may occur in the plant:

- the pump is not running completely the command it receives or there may be an additional pressure loss in the upper valve, which is assembled after the pump, and this determines the pump flow to be influenced by the fault, therefore it is no longer proportional to the control voltage;

$$
\Delta F_{p}=k_{p}\left(\Delta U_{C n}+\Delta U_{d}\right)
$$

Where $\Delta U_{C n}$ is pump control signal and $\Delta U_{d}$ the equivalent in the control signal of the actuator's fault.

- the modification of the flow section $S_{2}$ due either to a fluctuation of the flow system, by obturating the flowing section or additional pipeline breakings, which is equivalent to an increase of $S_{2}$; this determine that the discharge flow from the tank1in tank2 to be of the form:

$$
\Delta F_{2}=C \sqrt{L_{10}}\left(\Delta S_{2 n}+\Delta S_{2 d}\right)
$$

Where $\Delta S_{2 n}$ is the value of the section under normal functioning conditions and $\Delta S_{2 d}$ is equivalent value of the section, caused by the fault.

Under these circumstances, by introducing the faults specified in equations (6) and (7), replacing $\Delta U_{C}=\Delta U_{C n}+\Delta U_{d}$ and $\Delta S_{2}=\Delta S_{2 n}+\Delta S_{2 d}$ we obtain:

$$
\begin{aligned}
& \Delta \dot{x}_{1}=-\frac{C S_{20}}{2 A_{1} \sqrt{L_{10}}} \Delta x_{1}+\frac{k_{p}}{A_{1}}\left(\Delta U_{C}+\Delta U_{d}\right)-\frac{C \sqrt{L_{10}}}{A_{1}}\left(\Delta S_{2 n}+\Delta S_{2 d}\right) \\
& \Delta \dot{x}_{2}=\frac{C S_{20}}{2 A_{1} \sqrt{L_{10}}} \Delta x_{1}-\frac{k_{p}}{A_{1}}\left(\Delta U_{C}+\Delta U_{d}\right)+\frac{C \sqrt{L_{10}}}{A_{1}}\left(\Delta S_{2 n}+\Delta S_{2 d}\right)
\end{aligned}
$$

In equations (12) and (13) we have applied Laplace transform under zero initial conditions and using the transformations and the notes from the equations (8) and (9) it follows the operational form of the real process equations in which faults have been included.

Following the same steps to equations (12) and (13) as the relations (6) and (7) it results:

$$
x_{1 r}(s)=\frac{1}{T s+1}\left[\frac{2 x_{10} k_{p}}{F_{20}} \Delta U_{C n}(s)+\frac{2 x_{10} k_{p}}{F_{20}} \Delta U_{d}(s)-2 x_{10} \frac{\Delta S_{2 n}(s)}{S_{20}}-2 x_{10} \frac{\Delta S_{2 d}(s)}{S_{20}}\right]
$$




$$
x_{2 r}(s)=\frac{A_{1} / A_{2}}{T s+1}\left[-\frac{2 x_{10} k_{p}}{F_{20}} \Delta U_{C n}(s)-\frac{2 x_{1 r} k_{p}}{F_{20}} \Delta U_{d}(s)+2 x_{10} \frac{\Delta S_{2 n}(s)}{S_{20}}+2 x_{10} \frac{\Delta S_{2 d}(s)}{S_{20}}\right]
$$

Where: $\frac{C S_{20}}{2 A_{1} \sqrt{L_{10}}}=\frac{1}{T}$

From equations (8) and (9) it follows the process pattern, considered faultless $\Delta S_{2}=0$ :

$$
\begin{aligned}
& x_{1 m}(s)=\frac{1}{T s+1}\left[\frac{2 x_{10} k_{p}}{F_{20}} \Delta U_{C n}(s)-2 x_{10} \frac{\Delta S_{2 n}(s)}{S_{20}}\right] \\
& x_{2 m}(s)=\frac{1}{T s+1}\left[-\frac{2 x_{10} k_{p}}{F_{20}} \Delta U_{C n}(s)+2 x_{10} \frac{\Delta S_{2 n}(s)}{S_{20}}\right]
\end{aligned}
$$

We define the residues $r_{1}(\mathrm{~s})$ and $r_{2}(\mathrm{~s})$ [7]:

$$
\begin{aligned}
& r_{1}(s)=x_{1 r}(s)-x_{1 m}(s)=\frac{1}{(T s+1)} \frac{2 x_{10} k_{p}}{F_{20}} U_{d}(s)-\frac{1}{(T s+1)} 2 x_{10} \frac{\Delta S_{2 d}(s)}{S_{20}} \\
& r_{2}(s)=x_{2 r}(s)-x_{2 m}(s)=-\frac{1}{T s+1} \frac{2 x_{10} k_{p}}{F_{20}} \Delta U_{d}(s)+\frac{1}{T s+1} 2 x_{10} \frac{\Delta S_{2 d}(s)}{S_{20}(s)}
\end{aligned}
$$

From the equations (18) and (19) it can be seen that the residues $r_{1}$ and $r_{2}$ are both of influenced by the faults $\Delta S_{2 d}$ and $\Delta U_{d}$.

In Figure 2, the upper part is represented by the level control structure of the real process. This control structure allows adjusting the output of the process to the prescribed value even when the occurrence of a fault (the control system succeeds to compensate the fault). The command value of the model plant is function of the command value of the real controller and the output from the residual generator block, so in fault condition, the outputs of the model will show the real value of the level, when changing the command received from the controller. The output of the real plant $L_{1 r}$ and $L_{2 r}$ are compared to the output of the model plant $L_{1 m}$ and $L_{2 m}$ and the difference represents the input in the residual generator block. By simply monitoring of the residues it is possible to predict the change in behaviour of the system from normal condition.

Using the simulation scheme presented in Figure 2, the following experimental results have been obtained. In Figure 3 we can observe the process and the model outputs in normal conditions. In this case the residuals $r_{1}$ and $r_{2}$ are " 0 " as is shows in Figure 4.

In Figure 5and Figure 6 are represented the level for the model, respective the level for the real plant in fault condition, is considered the permanent fault $\Delta U_{d}=-0.2$ appeared at time $\mathrm{t}=40 \mathrm{~s}$. The residuals $r_{1}$ and $r_{2}$, represented in Figure 7 respective Figure 8, indicate the occurrence of the fault.

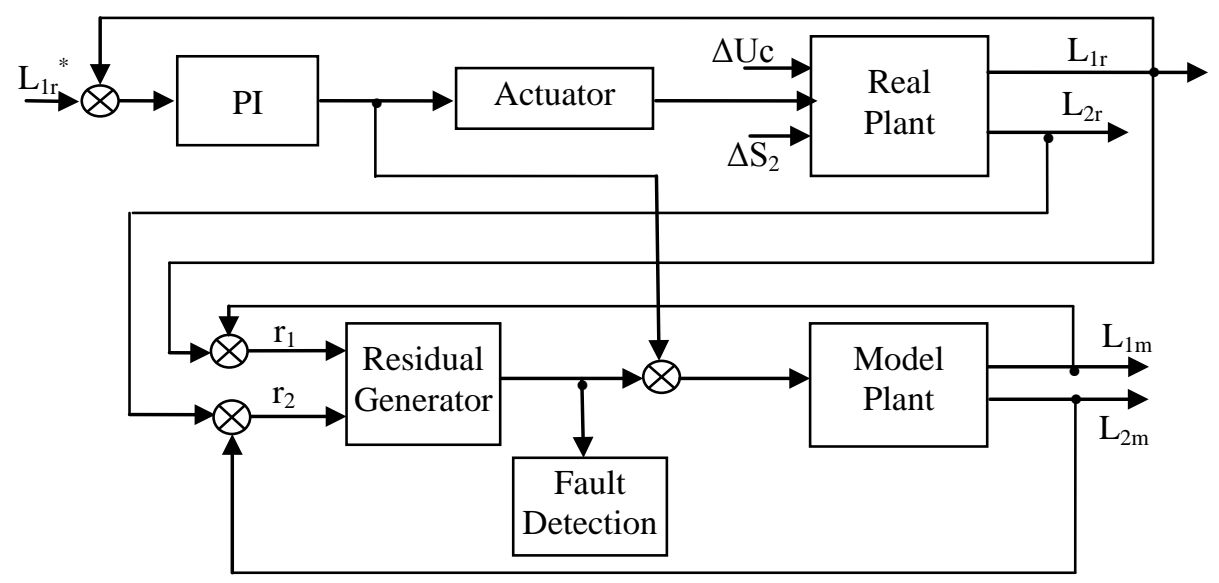

Figure 2: Fault detection and level control structure
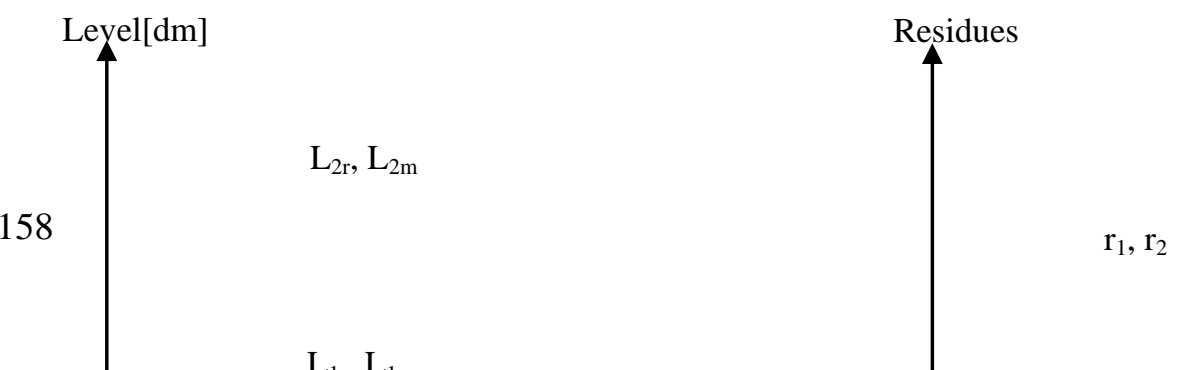


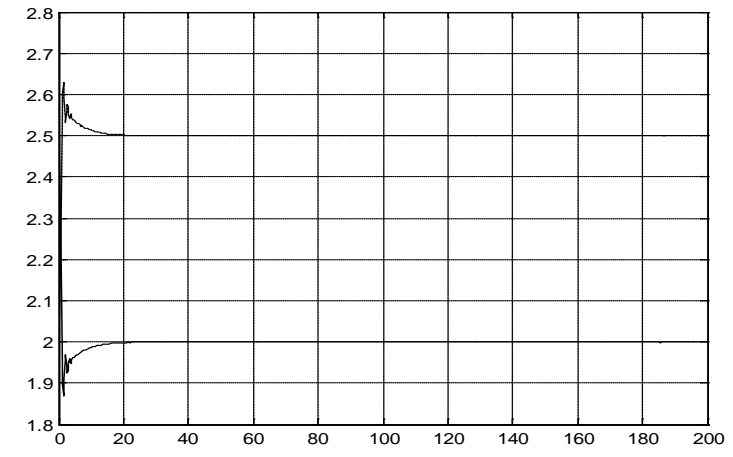

Figure 3: Process and model outputs in normal conditions

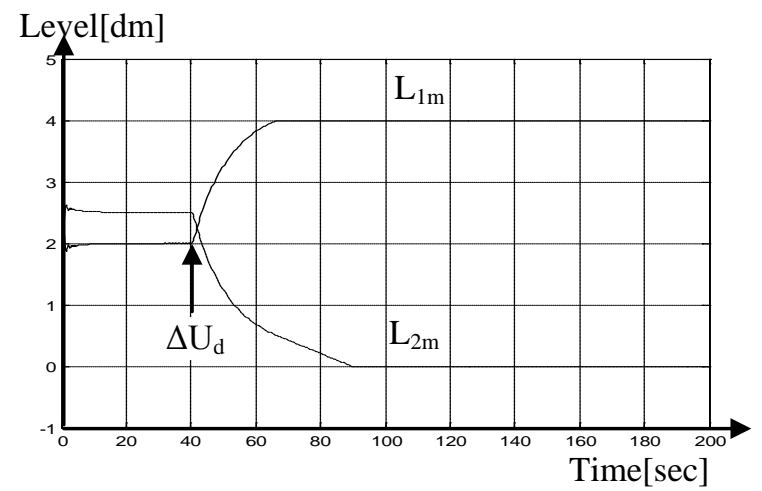

Figure 5: Level $L_{1 m}$ and $L_{2 m}$ for $\Delta U_{d}=-0.2$

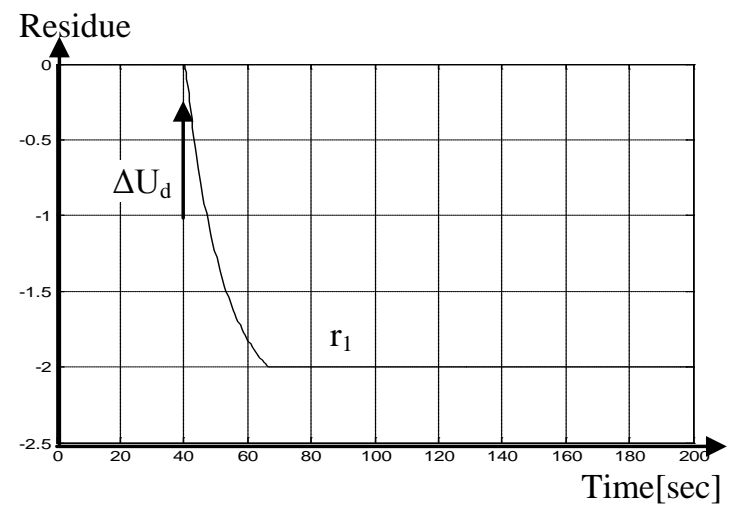

Figure 7: Residue $\boldsymbol{r}_{1}$ for permanent faul $\Delta U_{d}=-0.2$

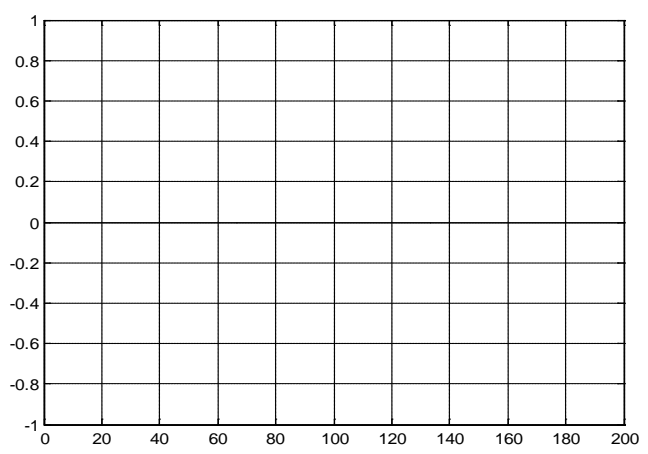

Figure 4: Residues in normal conditions

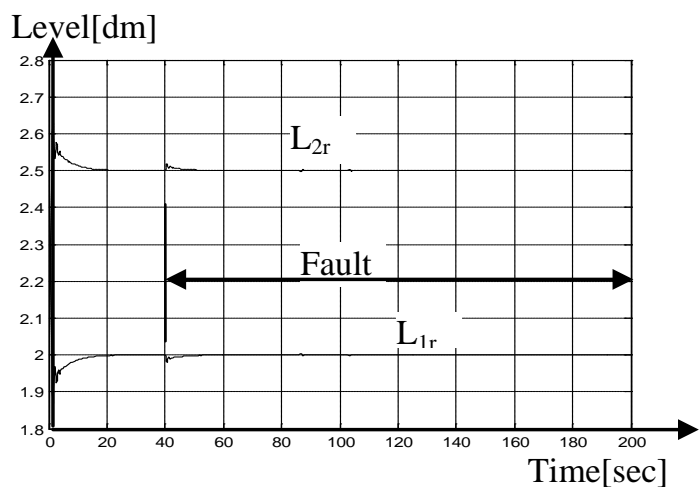

Figure 6: Level $L_{1 r}$ and $L_{2 r}$ for $\Delta U_{d}=-0.2$

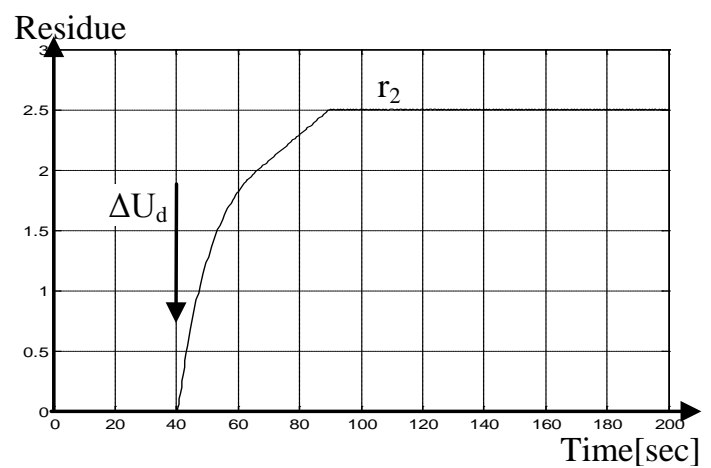

We consider that the fault $\Delta U_{d}=-0.2$ appears on the time $\mathrm{t}=40 \div 70 \mathrm{~s}$, in this case the level for the model is represented in Figure 9 and the level for the real plant is shown in Figure 10. Using the fault detection structure, the residues represented in Figure 11 and Figure 12 indicates the occurrence of the fault.

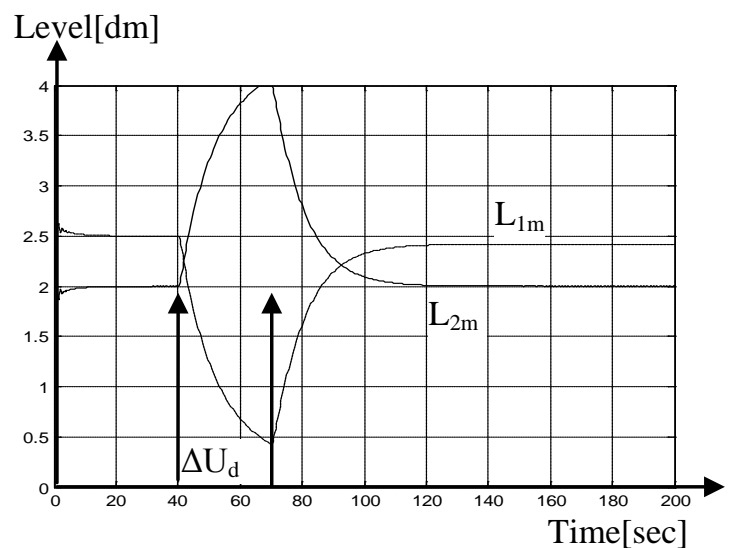

Figure 9: Level $L_{1 m}$ and $L_{2 m}$

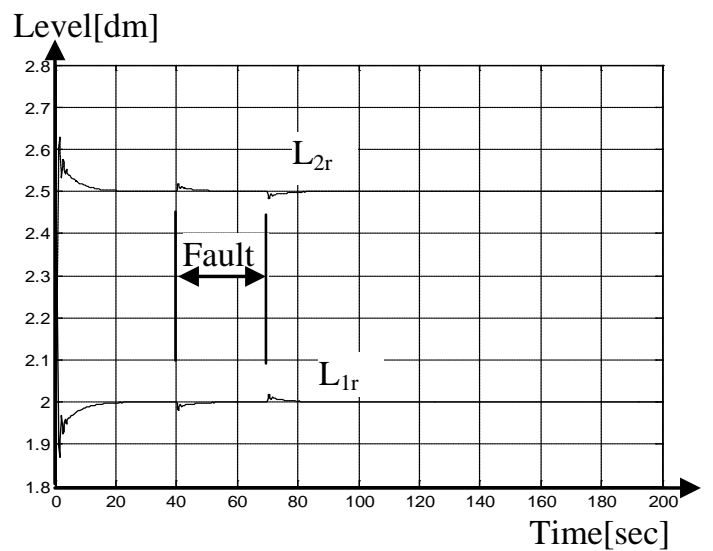

Figure 10: Level $L_{1 r}$ and $L_{2 r}$ 


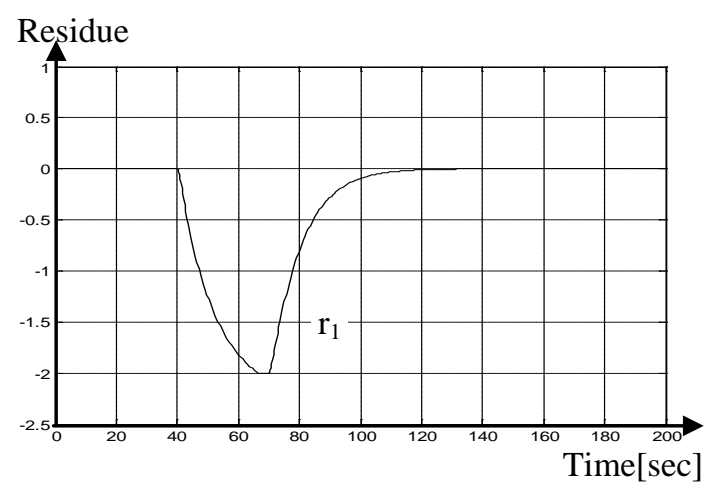

Figure 11: Residue $r_{1}$ for $\Delta U_{d}=-0.2$

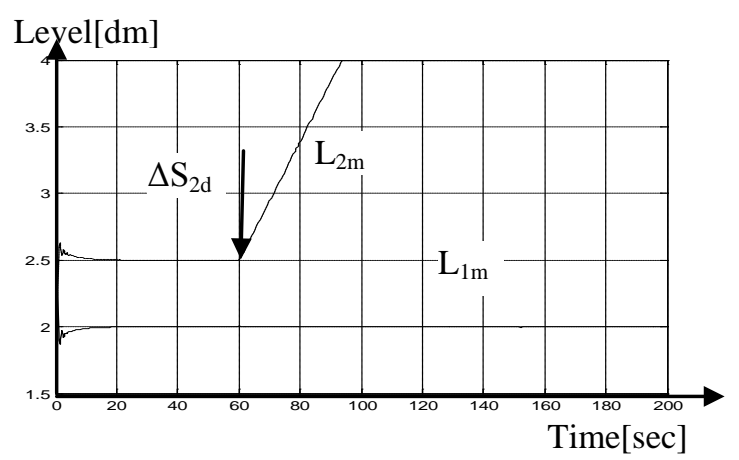

Figure 13: Level $L_{1 m}$ and $L_{2 m r}$ for $\Delta S_{2 \mathrm{~d}}=0.1$

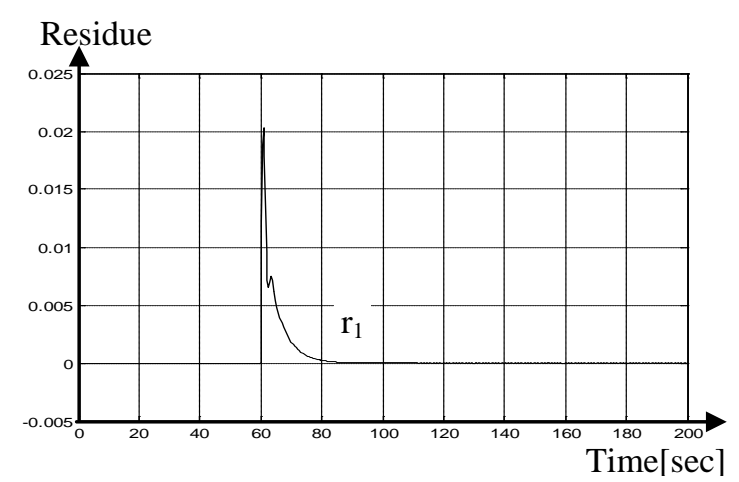

Figure 15: Residue $r_{1}$ for $\Delta S_{2 d}=0.1$

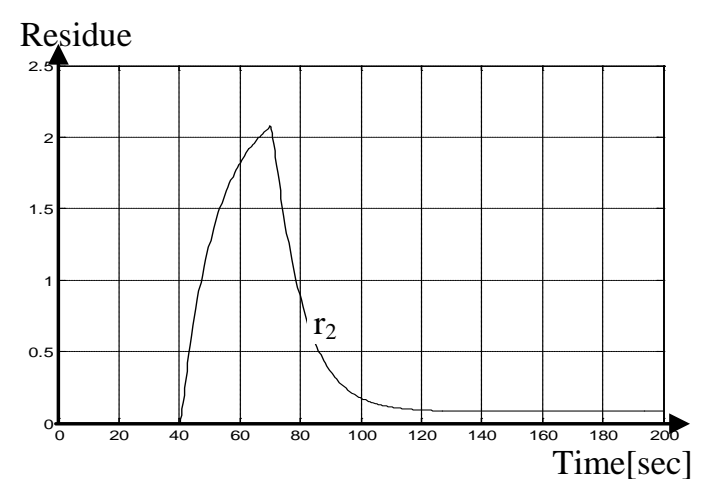

Figure 12: Residue $r_{2}$ for $\Delta U_{d}=-0.2$

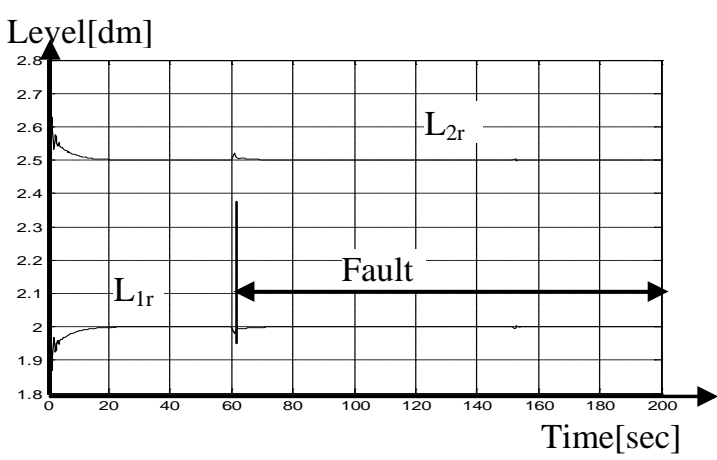

Figure 14: Level $L_{1 r}$ and $L_{2 r}$ for $\Delta S_{2 d}=0.1$

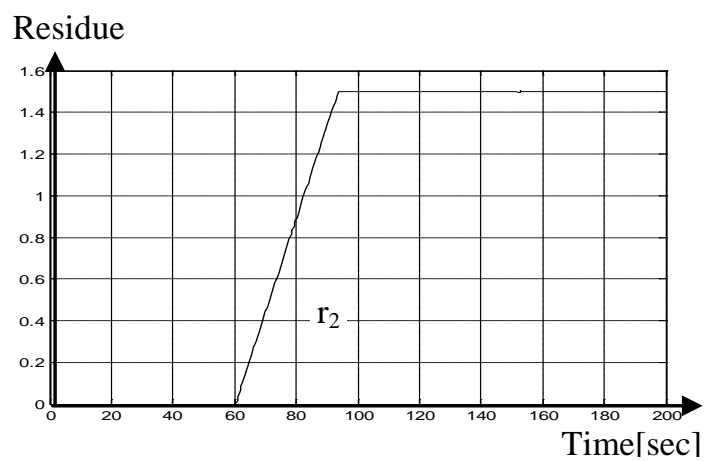

Figure 16: Residue $\boldsymbol{r}_{2}$ for $\Delta \mathrm{S}_{2 \mathrm{~d}}=\mathbf{0 . 1}$

In Figure 13 and Figure 14 are represented the level for the model, respective the level for the real plant in fault condition, is considered the permanent fault $\Delta S_{2 d}=0.1$ appeared at time $\mathrm{t}=60 \mathrm{~s}$. The residues represented in Figure 15 and Figure 16 indicates the occurrence of the fault.

\section{Conclusions}

In this paper a method for faults detection and localization using residual vectors was presented; the proposed method was theoretically developed and experimentally verified. It allowed detection and localization of two faults created in a real plant, including the detection of the cases when the control system succeeds to compensate the fault. This structure does not require supplementary equipments; it can be implemented as software complementary system on the existing monitoring digital control system from power plants. A series of case studies was realized regarding the possibilities for detection of the faults using residuals generator for the system. With the fault detection and localization scheme presented in this paper it can be detected the faults $\Delta U_{d}$ and $\Delta S_{2 d}$.

\section{Acknowledgment}

This work was supported by the strategic grant POSDRU/159/1.5/S/133255, Project ID 133255 (2014), co-financed by the European Social Fund within the Sectorial Operational Program Human Resources Development 2007 - 2013.

\section{References}


1. Isserman, R., Model-Based Fault Detection and Diagnosis - Status and Application, 16th IFAC Symposium on Automatic Control ACA 2004, Petersburg, Rusia, (2004).

2. Iancu, E., Vinatoru, M., Analytical method for fault detection and isolation in dynamic systems study case, Universitaria Craiova eds., Romania, (2003).

3. Zhang, H.-Y., Fault Detection, Supervision and Safety of Technical Processes, 6th IFAC Symposium on Fault Detection, Elsevier Science eds., (2006).

4. $\quad$ Gertler, J., Fault Detection and Diagnosis in Engineering Systems, Marcel Dekker eds., 1998.

5. Asokan, A., Sivakumar, D., Model based fault detection and diagnosis using structured residual approach in a multi-input multi-output system, Serbian Journal of Electrical Engineering, Vol. 4, No. 2, pp. 133-145, (2007).

6. Vinatoru, M., Iancu, E., Canureci, G., Maican, C., Automatic control of the industrial process - Design wizard and laboratory, vol. 2, Universitaria Craiova eds., Romania,(2008).

7. Canureci, G., Vinatoru, M., Maican, C., Fault detection and localization in dynamical systems, SITECH eds., Craiova, Romania, (2012). 